\title{
Rol de la Ortodoncia y la Ortopedia en el tratamiento de pacientes con traumatismos a nivel dentoalveolar. Reporte de casos
}

\section{Resumen}

Se presentan 2 casos clínicos de pacientes con traumatismos dentarios que avalan la importancia de un abordaje multidisciplinario a fin de optimizar su resolución.

Se resalta el rol del ortodoncista y el ortopedista dento-máxilo-facial en la prevención, atención adecuada de las diferentes situaciones que pueden presentarse en el transcurso de un traumatismo, y rehabilitación en las diferentes etapas de la dentición, optimizando resultados mediante una fluida interrelación con otros profesionales.

Se enfatiza la importancia de que, en la etapa diagnóstica y de planificación de los tratamientos, el profesional sea capaz de detectar secuelas de posibles traumatismos, que de pasar inadvertidos pueden complicar la resolución del caso.

Palabras Claves: Traumatismo dentoalveolar, Ortodoncia, Ortopedia.

Review article

\section{Orthodontics and Orthopedics' role in the treatment of patients with dentoalveoar trauma. Cases reports}

\begin{abstract}
Two clinical cases of dental trauma, showing the importance of the multidisciplinary approach in order to achieve an optimal solution, are presented.

Orthodontics and Dento-maxilo-facial Orthopedics' role is emphasized for dentoalveolar injury prevention, proper resolution of different situations in the evolution of a trauma, and opti-
\end{abstract}

mal treatment outcomes in different dental stages, by means of a fluid communication inside the dental team.

It is also extremey important in the diagnosis and treatment planning, that the practitioner be capable to find out possible unnoticed sequelae of trauma that could complicate the case resolution.

Key words: Dentoalveolar trauma, Orthodontics, Orthopedics. 


\section{O papel da Ortondontía é Ortopedia o pacientes con trauma dentoalveolar. Relato o casos}

\section{Resumo}

Nós apresentamos dois casos de pacientes com traumatismo dentário que suportam a importância de uma abordagem multidisciplinar para otimizar a sua resolução.

Ele destaca o papel do ortodontista e ortopedista dento-maxilo-facial na prevenção, o cuidado adequado das diferentes situações que podem ocorrer durante o trauma e reabilitação em diferentes estágios de dentição, otimizando resultados através da interação perfeita com outros profissionais.

Ela enfatiza a importância de que, na avaliação inicial e planejamento do tratamento, o profissional é capaz de detectar as seqüelas de trauma, o que pode complicar despercebido resolver o caso.

Palavras chave: Traumatismo dentoalveolar, Ortodontia, Ortopedia.

\section{Introducción}

El diagnóstico de las secuelas traumáticas y las posibilidades de solución de las mismas, adquieren relevancia dentro de la odontología debido al alto índice de prevalencia así como por sus consecuencias estéticas y funcionales, ya que como varios estudios lo evidencian, un $25 \%$ de los adultos presentan signos de traumatismos dento-alveolares. ${ }^{1,2}$

La Ortodoncia y la Ortopedia Dento-máxilofacial tienen aportes valiosos en relación a la prevención y al tratamiento de estos casos. ${ }^{2}$
Cuando se produce un traumatismo dentario, es de importancia la realización de un tratamiento de urgencia adecuado para lograr el éxito de la evolución posterior. ${ }^{2,3}$ Una vez realizado el mismo, el rol del ortodoncista en la planificación del tratamiento con una visión multidisciplinaria del caso, optimiza los resultados. ${ }^{2}$ De esta manera se hacen necesarios, al momento de tomar decisiones terapéuticas, los aportes del odontólogo general, el odontopediatra, el endodoncista, el ortodoncista, y algunas veces del cirujano y el periodoncista, dependiendo de la complejidad del caso clínico.

Una vez solucionada la urgencia, es de importancia la realización de un completo diagnóstico clínico y paraclínico del caso, que teniendo en cuenta la edad cronológica y biológica, permita evaluar la oportunidad de realizar un tratamiento de Ortodoncia u Ortopedia Funcional. ${ }^{2}$

Las diferentes etapas del desarrollo de los maxilares y de la evolución madurativa y eruptiva dentaria van a delinear conductas distintas, que se adecuen a cada situación.

El ortodoncista también juega un rol fundamental en la prevención de los traumatismos, así como en la pesquisa de las posibles consecuencias mediatas de los mismos, antes de la resolución ortodóncica de un caso. ${ }^{2,3}$

\section{Prevalencia de traumatismos dentarios}

Luciane Ribeiro de Rezende, haciendo referencia a Lacerda, define traumatismo como "una 
lesión de extensión, intensidad y gravedad variable, de origen accidental o intencional, causada por fuerzas que actúan en el órgano dentario como resultado de accidentes, golpes, etc." ${ }^{4}$

Los traumatismos en la región oral ocurren con gran frecuencia en edad pre-escolar, escolar y en adultos jóvenes y constituyen el 5\% del total de las lesiones presentadas por los individuos que solicitan asistencia. ${ }^{1}$ Los picos de máxima frecuencia de traumatismos en la dentición permanente, en niños escolarizados, se encuentra entre los nueve y los diez años. ${ }^{1,5,6}$ La prevalencia de los mismos oscila entre un $2,4 \%$ y un $58 \%$ y es mayor en varones que en niñas en una relación de 1,3 - 2,3 a 1 . Hoy, con la inserción cada vez mayor de niñas en deportes violentos, ha cambiado notoriamente la relación de prevalencia entre niños y niñas. ${ }^{1,5,6}$

En etapa de dentición temporaria, la prevalencia varía entre un $4 \%$ y un $33 \%$ y la diferencia entre

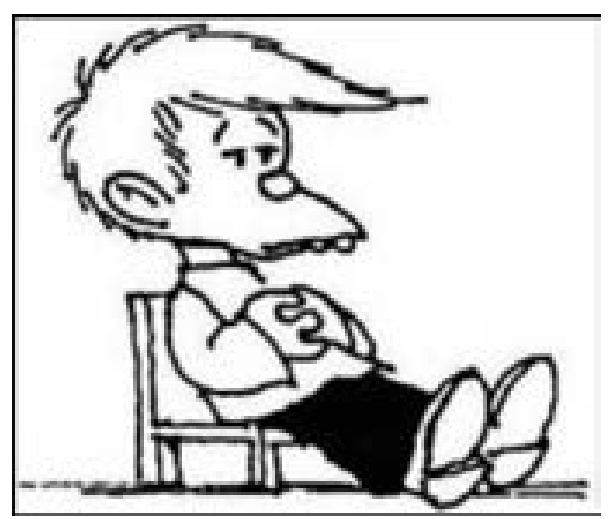

Figura 1. Felipe. Personaje de la tira humorística "Mafal$d a^{\prime \prime}$ de Quino, representa un típico perfil pro-trauma. géneros disminuye a una relación de $0,9-1,3$ a $1 .{ }^{1,7}$

La variación tan grande entre las cifras dadas por los diferentes estudios parece asociarse a múltiples factores, como el método de registro, lugar del estudio, población analizada, edades y país. ${ }^{1,7}$

\section{Factores Predisponentes}

Uno de los factores que predispone a los traumatismos dentoalveolares es la protrusión de incisivos superiores, con un resalte horizontal aumentado, agregado a una incompetencia labial que deja sin protección a estas piezas, expuestas a golpes relacionados con juegos, deportes (borde de la pileta de natación, palos de jockey, etc.), y demás posibles traumatismos. ${ }^{1}$ Malgrem $\mathrm{O}$. señala que de acuerdo a estudios, un aumento del resalte horizontal de los incisivos de 0-3 mm a 3-6 $\mathrm{mm}$, duplica la extensión de lesiones dentarias traumáticas, y con un overjet mayor a 6 mm la severidad se triplica. ${ }^{8}$ Fig. 1, 2, 3.

\section{Traumatismos en dentición decidua}

Esta etapa del niño que se extiende desde la erupción de sus primeras piezas hasta los $4 \mathrm{o}$ 5 años de vida, es muy rica en cambios en lo relacionado al desarrollo de los maxilares y la oclusión. Cualquier modificación que impida el
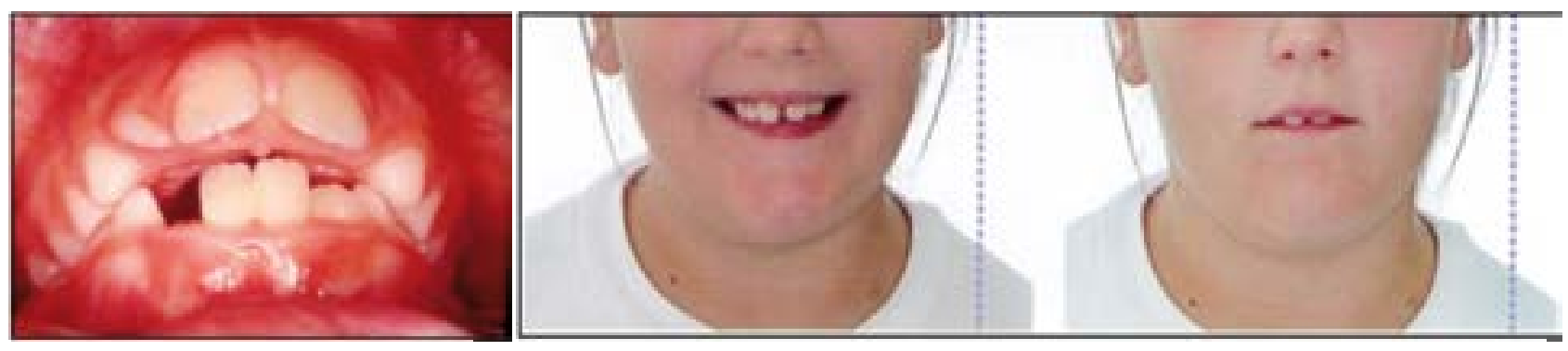

Figuras 2 y 3. Pacientes en etapa de dentición mixta que presentan overjet aumentado, con proinclinación de incisivos e incompetencia labial que deja a los incisivos superiores expuestos a trauma. 
correcto crecimiento incidirá directamente en el sistema estomatognático del adulto. La frecuencia de los traumatismos en esta etapa es alta. Varios estudios evidencian que 1 de cada 3 niños en fase de dentición decidua sufren lesiones traumáticas de la región bucal. ${ }^{4,9}$

Es frecuente a estas edades y con los primeros pasos, la luxación e incluso la avulsión traumática de los incisivos, fundamentalmente los superiores. Las luxaciones intrusivas ocurren con más frecuencia que las fracturas coronarias en estas etapas, debido entre otras cosas, a la presencia de un proceso alveolar más esponjoso, a que las coronas son de menor tamaño en relación a la raíz, a que en general las raíces presentan acortamiento por la rizálisis fisiológica y a que el ligamento periodontal es más laxo.,4,10

Vigilar la evolución del proceso de traumatismo es importante para conocer con anticipación la posible afección de los gérmenes de los permanentes sucesores, los cambios del trayecto eruptivo de los mismos, y evitar, en casos de pérdida total de la pieza decidua, la disminución de la longitud de la arcada. ${ }^{11,12}$

En casos de traumatismos en esta etapa, antes de instalar medidas terapéuticas adecuadas al caso, se deben tener en cuenta, fundamentalmente, las relaciones esqueléticas y dentarias para poder guiar el sistema estomatognático hacia un correcto desarrollo de maxilares y una adecuada oclusión. ${ }^{2}$

El conocimiento del desarrollo normal del sistema estomatognático y las diferentes terapéuticas a emplear son importantes para no impedir o alterar el correcto desarrollo del mismo a través del uso de dispositivos inadecuados, por ejemplo, interfiriendo en la erupción de los dientes o con el crecimiento de los maxilares. ${ }^{2,8,12}$. Las in- terconsultas frecuentes entre el odontopediatra y el ortodoncista son fundamentales en todas las etapas del tratamiento.

\section{Traumatismos en dentición mixta}

Muchos autores coinciden en que el mayor número de traumatismos dentarios se da durante esta etapa del niño. ${ }^{1,2,3,4,7,10}$ Los estudios confirman que los deportes de contacto son los principales eventos de lesiones dentales traumáticas. La prevalencia de las lesiones dentales durante la práctica deportiva llega a ser hasta de un $45 \%$, siendo las más frecuentes las luxaciones y fracturas dento-alveolares. ${ }^{1,7}$ La inclinación normalmente mayor de los incisivos permanentes comparada con la de los temporarios, en relación a la cresta ósea, determina una mayor propensión a la afección de dichas piezas a través de un golpe. ${ }^{1,8}$

Siendo las disgnacias factores indudablemente predisponentes que inciden en la ocurrencia de los traumatismos dentarios, es claro que el ortodoncista está llamado a ejercer su función tomando las medidas preventivas inmediatas del caso, e incluso, mejorando las relaciones maxilares y dentarias a través de tratamiento ortopédico u ortodóncico, de acuerdo a lo que sea necesario. En los niños que presentan Clase II, 1ra. división, involucrando una relación distal de los maxilares, y que se encuentran en etapas de crecimiento, será tarea del ortodoncista-ortopedista, redireccionar el crecimiento de los maxilares lo máximo que el biotipo del paciente lo permita, guiando la evolución hacia una eugnacia.

La labor educativa del odontólogo y el uso de protectores bucales son las medidas preventivas más eficientes en pacientes que practican deportes, especialmente aquellos de contacto físico o que impliquen caídas y golpes. Esto es 

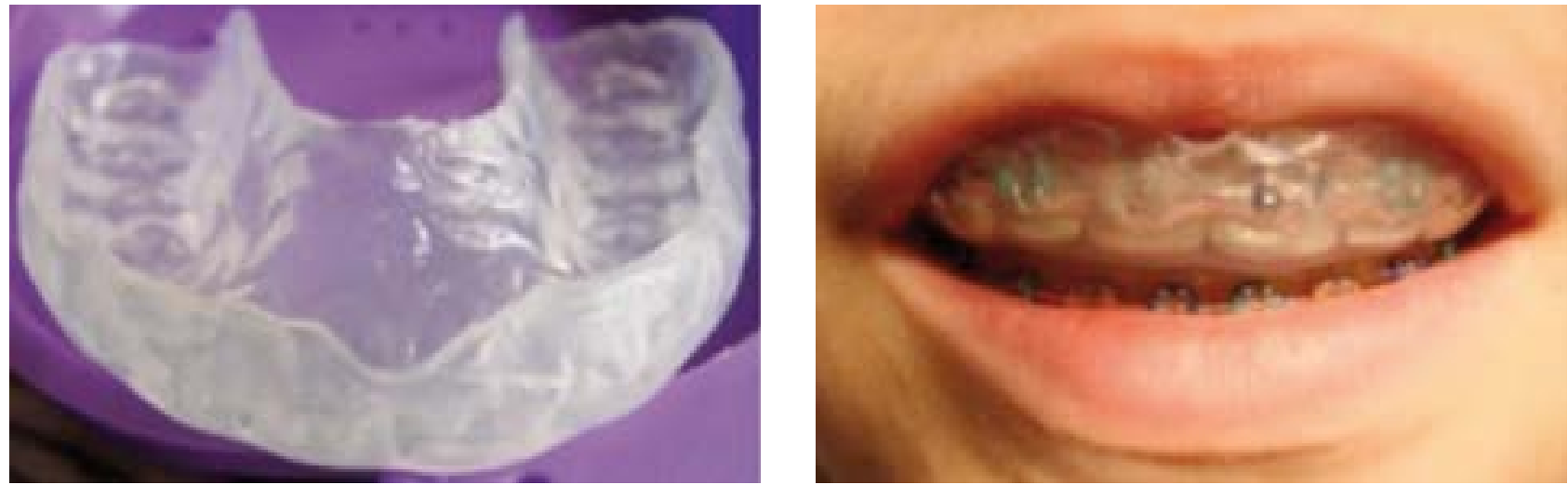

Figuras 4 y 5. Protector bucal estampado para paciente en tratamiento de Ortodoncia.

así para todos los individuos, pero fundamental en aquellos que presentan proinclinación de incisivos superiores. ${ }^{1,2}$ Los protectores bucales pueden ser de muchos tipos y materiales., ${ }^{413}$ Figs. 4 y 5.

Algunos de los requisitos que deben cumplir los Protectores Bucales son:

1. Prolijidad en la confección y correcto acabado, de manera de no lesionar tejidos blandos durante su uso y que el mismo no provoque inconvenientes.

2. Cubrir todas las superficies dentarias a proteger, manteniendo una relación de arcadas lo más amplia y correcta posible, de manera de impedir cualquier trastorno de la articulación témporomandibular durante su uso, fundamentalmente por impactos recibidos a nivel del mentón mientras el mismo se encuentra en boca.

3. Ser de fácil construcción y adaptación en lo posible. De preferencia, construidos a medida,permitir el habla y la respiración, siendo cómodos en su uso.

4. Ser elásticos.

5. Ser resistentes.
En todos los casos, antes de comenzar un tratamiento de Ortodoncia, se deberán estudiar en forma clínica y radiográfica posibles secuelas de traumatismos que han pasado en forma inadvertida y que pueden llegar a resultar en posibles contratiempos durante el tratamiento. ${ }^{2}$

Después de superado el tratamiento de emergencia, se planifican las estrategias de tratamiento para solucionar las secuelas del trauma junto con el equipo de trabajo donde el ortodoncista juega un papel importante. ${ }^{2}$ El aporte del endodoncista, en casos de graves compromisos pulpares que escapen a la habilidad del odontólogo general, es fundamental para dar una solución óptima al caso. Los periodos de observación con controles clínicos y radiográficos que se deben usualmente guardar luego del trauma, son de 1 año en caso de una lesión leve (subluxación, concusión) y de lesión moderada a severa (fractura de corona, extrusión, intrusión, reimplantación); 5 años en casos de fracturas radiculares; y en dientes con tratamiento endodóncico, se sugiere posponer el tratamiento ortodóncico hasta observar evidencia radiográfica de curación. ${ }^{5,12,14,15}$

Es importante conocer que cualquier fuerza aplicada a un diente traumatizado, es un riesgo que se suma a la respuesta del periodonto y 
la pulpa del mismo. Sin embargo, el manejo de fuerzas de baja intensidad, luego del probable tratamiento de conducto y periodo de observación aconsejado por el endodoncista, logra llevar a la posición adecuada a las piezas injuriadas, con un mínimo de daño, devolviéndoles una función prácticamente normal. ${ }^{8}$

En casos de subluxaciones, luxaciones extrusivas y avulsiones traumáticas el período de inmovilización de las piezas que se debe considerar es de 2 semanas: 4 semanas en casos de luxaciones laterales, fracturas radiculares del tercio medio y fractura alveolar; y 4 meses en fracturas radiculares del tercio cervical. Se prefieren las férulas semirígidas a las rígidas. permitiendo así pequeños movimientos fisiológicos que aseguran una adecuada reparación de los tejidos periodontales, pues se favorece la revascularización. 2,5,15,16

En los casos de luxación, en los que el diente presente necrosis y/o reabsorción radicular inflamatoria, éste deberá ser tratado endodóncicamente antes del tratamiento de Ortodoncia, y siempre deberá ser controlado radiográficamente en forma periódica para conocer la evolución que determinarán la conducta más adecuada.,

En las intrusiones se tendrá en cuenta el grado de apexogénesis de la pieza injuriada. En los casos de dientes inmaduros, debe tomarse una conducta expectante de dos a tres semanas en espera de una erupción espontánea. Si no lo hace, la extrusión ortodóncica está indicada. ${ }^{5,8}$ Fig. 6.

Cuando se trata de un diente que ha completado la maduración radicular, el tratamiento endodóncico profiláctico es recomedado ${ }^{8}$. Si la intrusión no es acompañada de fractura de paredes alveolares severas, puede realizarse la extrusión ortodóncica del mismo, a través de fuerzas de baja intensidad y constantes, durante un período breve que debería oscilar entre 3 y 6 semanas, (antes que la anquilosis tenga lugar) y darle una ferulización luego del movimiento que permita la consolidación de todo el aparato de soporte. ${ }^{5}$ Debe haber un riguroso seguimiento radiográfico para conocer la respuesta de la pulpa y del aparato de soporte, ya que es frecuente observar en estos casos reabsorciones internas y externas de la raíz. ${ }^{8,17}$ Fig. 7.

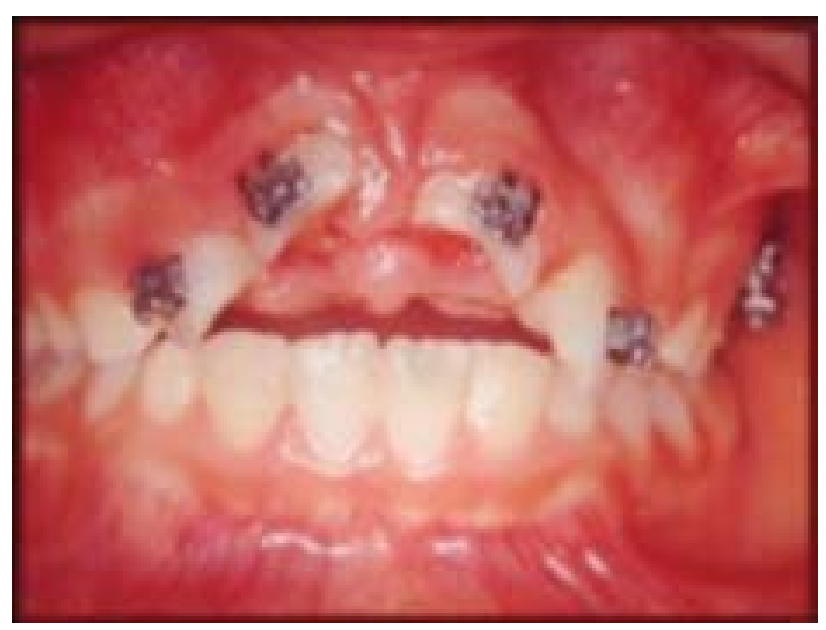

Figura 6. Paciente en dentición mixta que sufrió traumatismo intrusivo de los cuatro incisivos superiores. Pasado el momento de emergencia, con los brackets colocados para realizar el tratamiento de extrusión y alineación dentaria con fuerzas de baja intensidad.

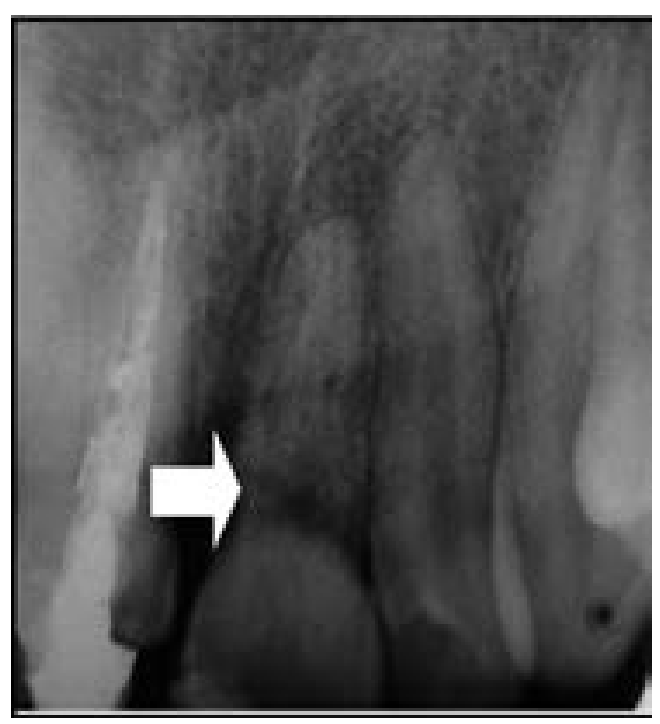

Figura 7. Reabsorciones internas y externas en incisivos centrales traumatizados. Hallazgo previo al tratamiento de Ortodoncia. 
En las piezas con fractura radicular, habrá que considerar la localización de la fractura y el tipo de curación que puede haberse dado a través de tejido calcificado (de mejor pronóstico) o por interposición de tejido conectivo. ${ }^{8,18}$ Cuanto más apical sea la fractura, más favorable será el pronóstico del movimiento ortodóncico. ${ }^{8}$

Cuando hay pérdidas de piezas, la decisión de plan de tratamiento se tomará, como en todos los casos, luego de un exhaustivo diagnóstico. No existen cánones estrictos de cómo proceder cerrando espacios o sustituyendo al diente perdido, porque eso depende de muchos factores (discrepancia dento-alveolar negativa, relación intermaxilar, posibilidad de crecimiento residual, entre otros), que deben tenerse en cuenta y que el ortodoncista deberá evaluar antes de comenzar a resolver el caso. ${ }^{2,8}$

Los dientes que luego de la avulsión traumática tuvieron la oportunidad de ser reimplantados pueden responder manteniendo un periodonto sano o anquilosándose, lo que se evaluará radiográficamente antes de abordar el tratamiento ${ }^{8,10}$, En el primer caso el movimiento ortodóncico es posible en forma normal. En el segundo, habrá que tomar decisiones de mantenerlo o extraerlo según los requerimientos del tratamiento, pero no es posible trasladarlos a través del uso de fuerzas ortodóncicas ya que no hay periodonto que responda biológicamente a las mismas. Es importante que en caso de avulsión, se tomen en cuenta todas las medidas necesarias para que la pérdida de hueso en la cirugía sea la menor posible. $^{8}$

\section{Traumatismos en dentición permanente}

Todas las consideraciones de la sección anterior son válidas para la dentición permanente. La resolución de los casos estará diferenciada noto- riamente en que cuando la dentición permanente ya está establecida, el crecimiento y desarrollo de los maxilares está prácticamente en su final, de manera que ya no se puede considerar como una variable que incida en la planificación del tratamiento.

Antes de comenzar un tratamiento de ortodoncia, siempre se debe cuidar: la forma de las raíces dentarias y la posibilidad de reabsorciones radiculares como consecuencia de antiguos traumatismos. ${ }^{8,19}$

Ya instalado el tratamiento, se deben realizar controles radiográficos periódicos para verificar si se presentan o aumenta la reabsorción radicular de los dientes con historia de traumatismos. El primer control se aconseja entre los 6 y 9 meses del comienzo. Si aparecieran signos de reabsorción, están indicados controles cada dos meses y en muchos casos se sugiere una suspensión del tratamiento de Ortodoncia por un período de 3 meses, reduciendo de esta manera el riesgo a reabsorciones severas. ${ }^{2,8}$

\section{Relato de casos}

Caso 1 - Paciente M.A.

Edad: 12 a. $5 \mathrm{~m}$.

Sexo: masculino.

Biotipo: mesofacial.

Motivo de consulta: Incisivos protruídos.

Antecedentes personales: Traumatismo dentario con fractura de borde incisal de pieza 11.

\section{Síntesis diagnóstica}

- Evaluación esquelética: Relación distal de maxilares. Birretrusión. 
- Evaluación dentaria: Clase II molar y canina (más severa del lado izquierdo). Overjet aumentado. No coincidencia de líneas medias dentarias. Rotación de piezas 16 y 26. Fractura coronaria de pieza 11, con compromiso de esmalte y dentina.

- Evaluación funcional: Respiración bucal. (Hipertrofia de adenoides). Deglución con interposición labial.

- Evaluación estética: Leve asimetría. Perfil convexo. Ángulo naso-labial abierto.

- Evaluación periodontal: Gingivitis incipiente en algunos sectores.
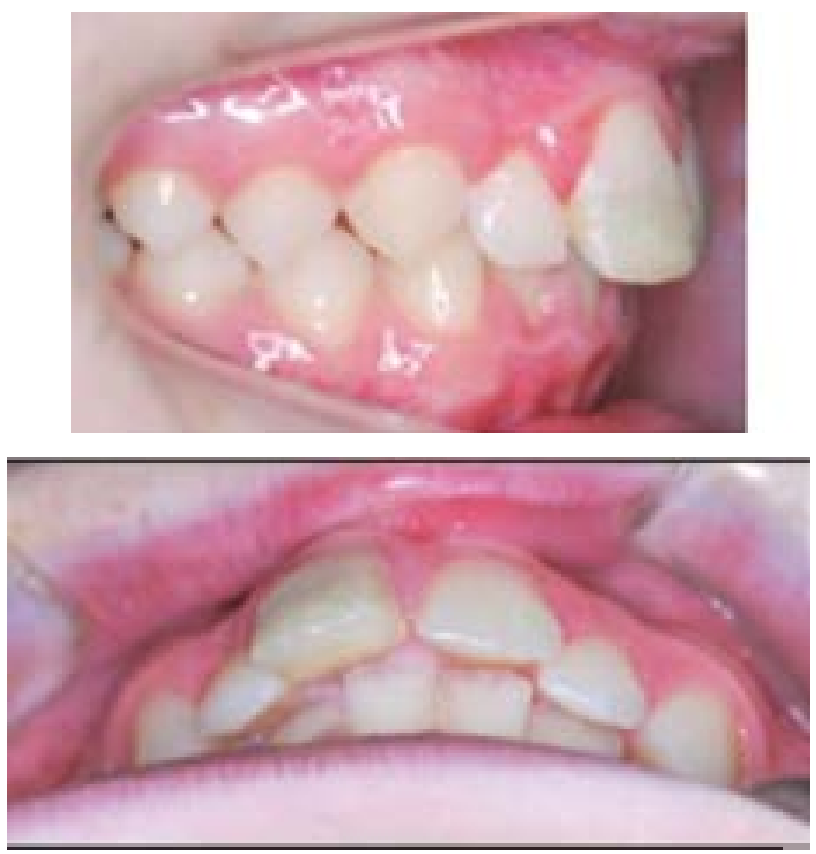

- Evaluación endodóncica: Pieza con vitalidad pulpar.

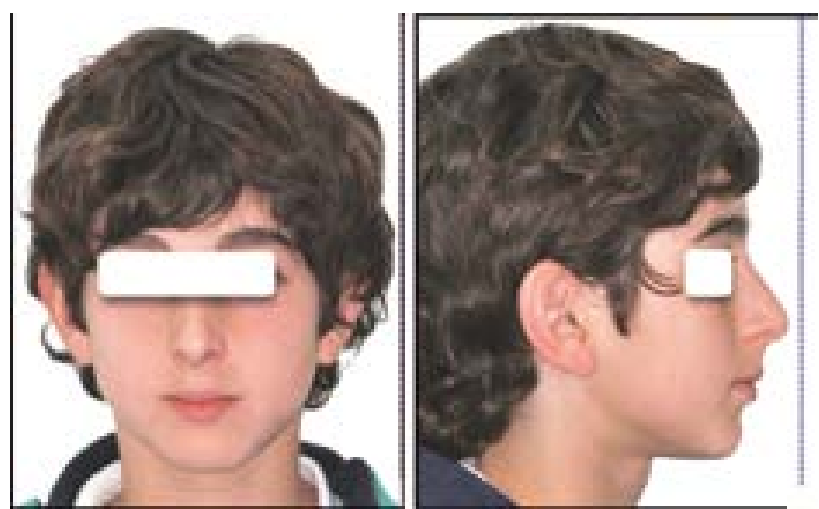

Figuras 8 y 9. Paciente M.A. Fotos frente y perfil iniciales.
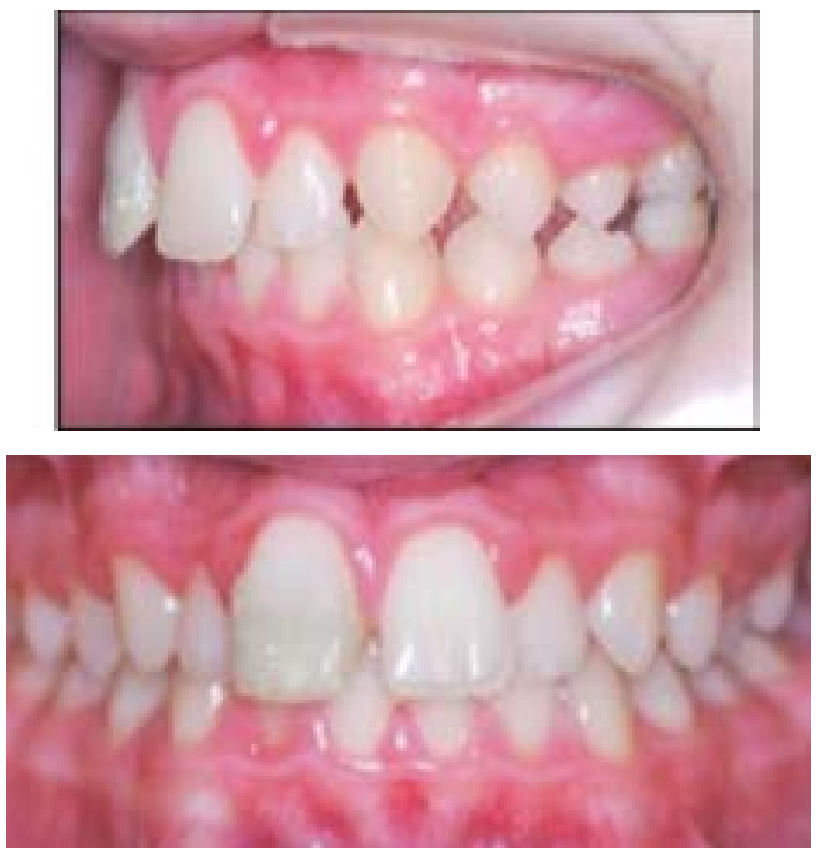

Figuras 10 a 13. Fotos intrabucales iniciales frente, perfiles derecho e izquierdo y overjet.
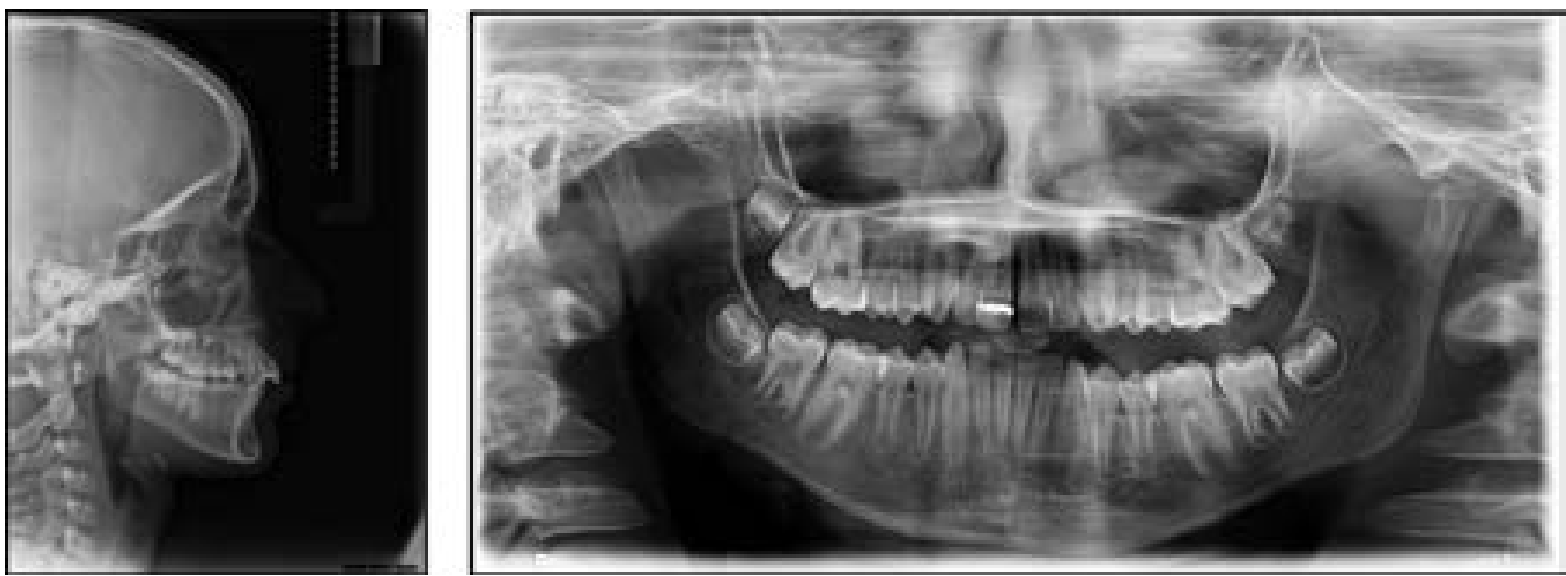

Figuras 14 y 15. Telerradiografía y Ortopantomografía iniciales. 


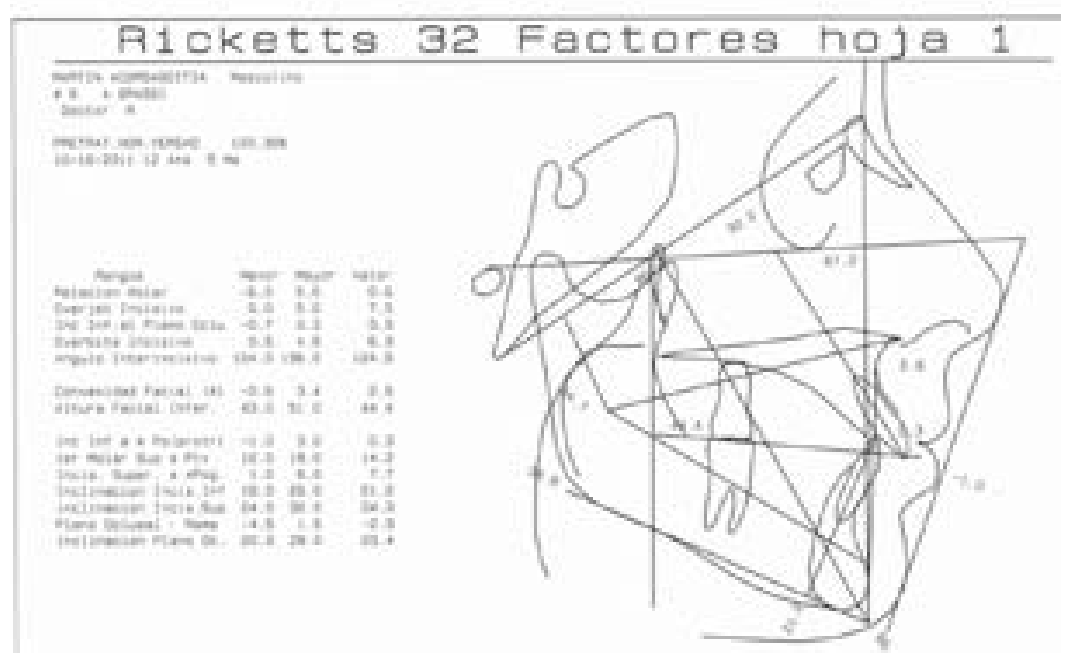

Figura 16. Cefalograma de Ricketts.

\section{$\underline{\text { Plan de tratamiento }}$}

Médico - Odontológicos: Derivación a otorrinolaringólogo para la evaluación de permeabilidad de vías respiratorias. Reeducación de la deglución.

Odontopediatría: Al momento de la fractura de pieza 11, se realizó colocación de Hidróxido de Calcio fraguable y técnica de collage, ya que se recuperó resto incisal. Control clínico y radiográfico de la pieza por un período de un año antes del comienzo del tratamiento de Ortodoncia.

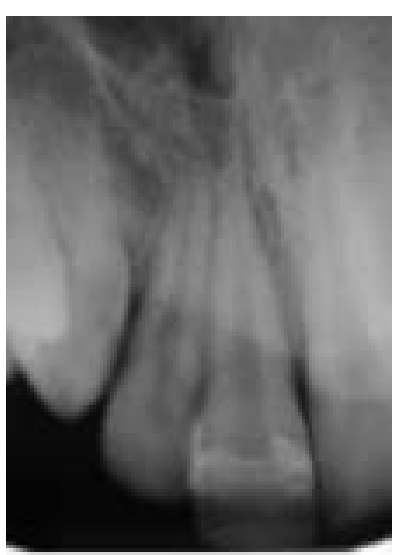

Figura 17. Radiografía del 18/08/10, inmediata a la fractura y a la restauración.

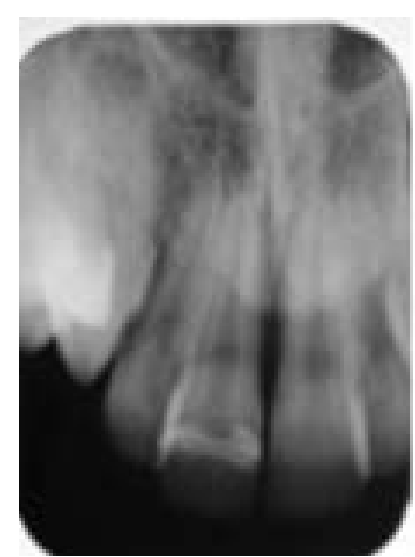

Figura 18. Radiografía de 14/11/11, al año del traumatismo y previo a la colocación de aparatología fija.
Profilaxis y enseñanza de cepillado.

Ortodóncicos: Rotación de piezas 16 y 26. Intrusión (con fuerzas de muy baja intensidad) y retrusión de sector ántero-superior, para disminuir overjet. Intrusión leve del sector ántero inferior. Lograr llave canina. Cuidar estética, fundamentalmente del perfil.

Medio terapéutico:

- Aparatología fija, multibracket, Técnica de Arco Recto, prescripción Roth 0.022. Gomas intermaxilares de Clase II. 

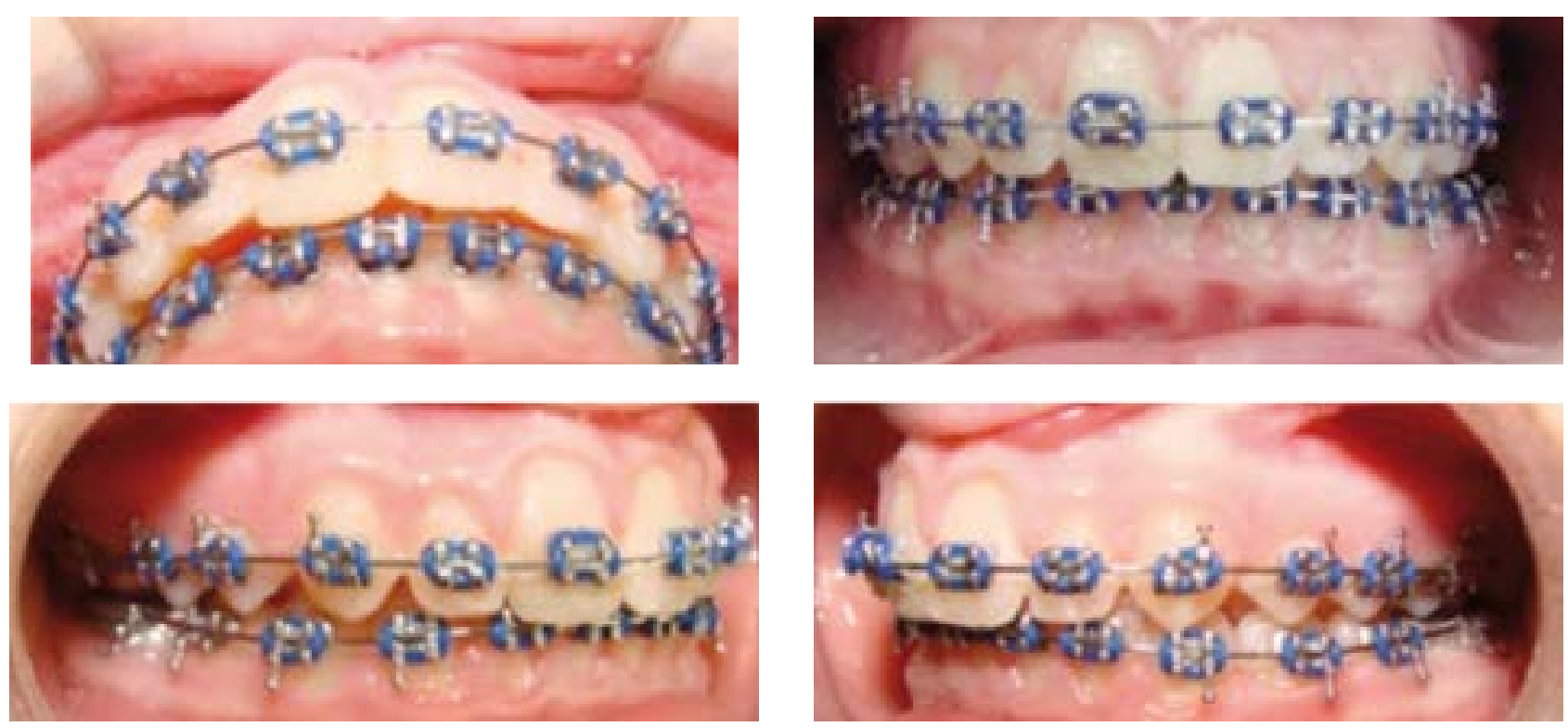

Figuras 20 a 23. Fotografía bucal del paciente con brackets en etapa de alineación y nivelación.

Caso 2 - Paciente B.B.

Edad: 8 años 10meses.

Sexo: masculino.

Biotipo: braquifacial severo.

Motivo de consulta: Derivado por la fonoaudióloga que trata reeducación de la deglución, por protrusión de incisivos superiores.

Antecedentes personales: Paciente sano, con desarrollo acorde a su edad, y condiciones socioeconómicas favorables.

- Evaluación esquelética: Relación distal de maxilares, fundamentalmente a responsabilidad de protrusión de maxilar superior.

- Evaluación dentaria: Dentición mixta. Clase I molar. Relación canina normal. Sobrepase horizontal aumentado. No coincidencia de líneas medias dentarias. Sobrepase vertical aumentado, a predominio de ex- trusión de incisivos inferiores. Rotación de piezas 16 y 26. Diastemas múltiples, primordialmente en arcada superior. Una semana antes de instalar terapéutica funcional, el paciente sufrió traumatismo dentario, presentando como consecuencia del mismo fractura coronaria no complicada de pieza 11, con compromiso de esmalte y dentina.

- Evaluación funcional: Deglución con interposición labial.

- Evaluación estética: Muy leve asimetría. Perfil convexo. Ángulo naso-labial abierto. Surco labio - mentoniano muy marcado.

- Evaluación periodontal: Gingivitis incipiente en algunos sectores a causa de la placa bacteriana existente.

- Evaluación endodóncica: Fractura no complicada de esmalte y dentina coronaria de pieza 11 , con vitalidad pulpar. 


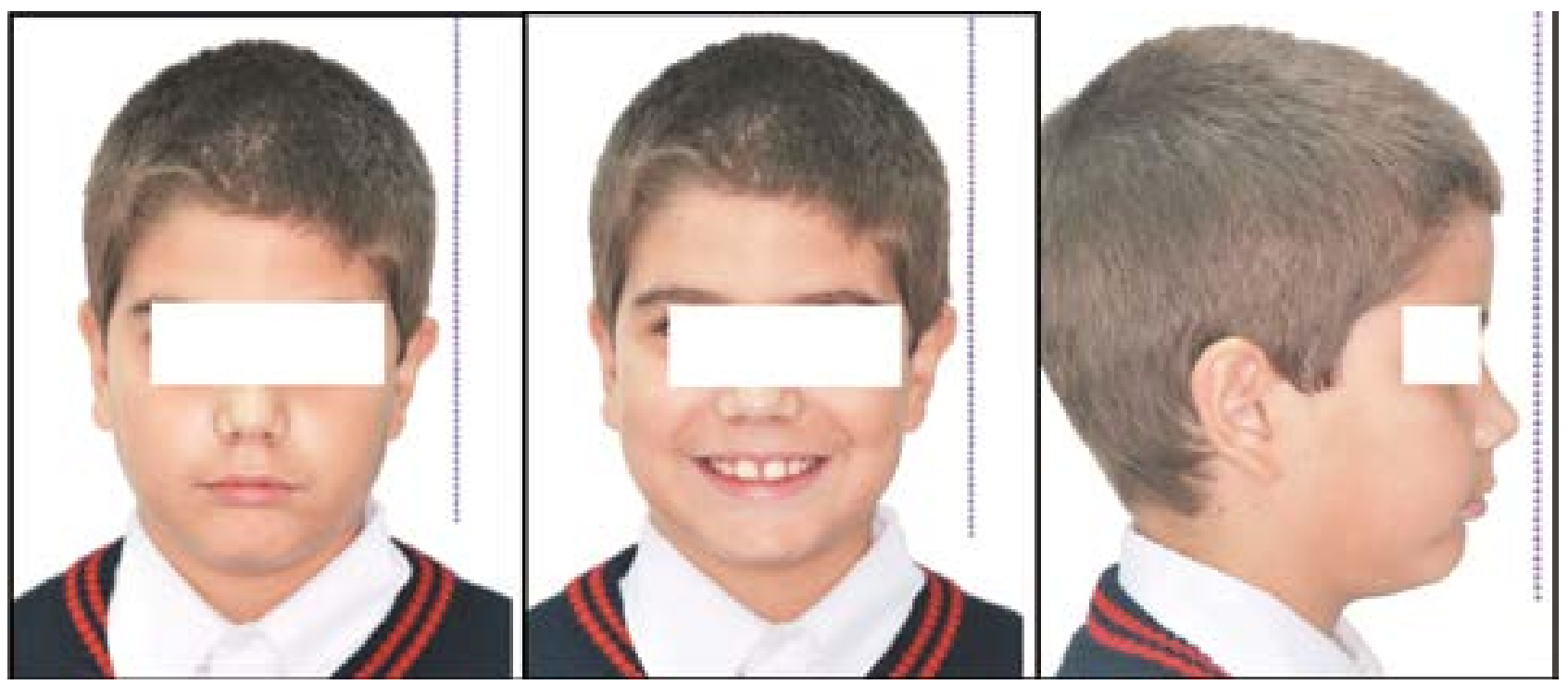

Figuras 24 a 26. Paciente B.B. Fotografías frente reposo y sonrisa y perfil de cara.
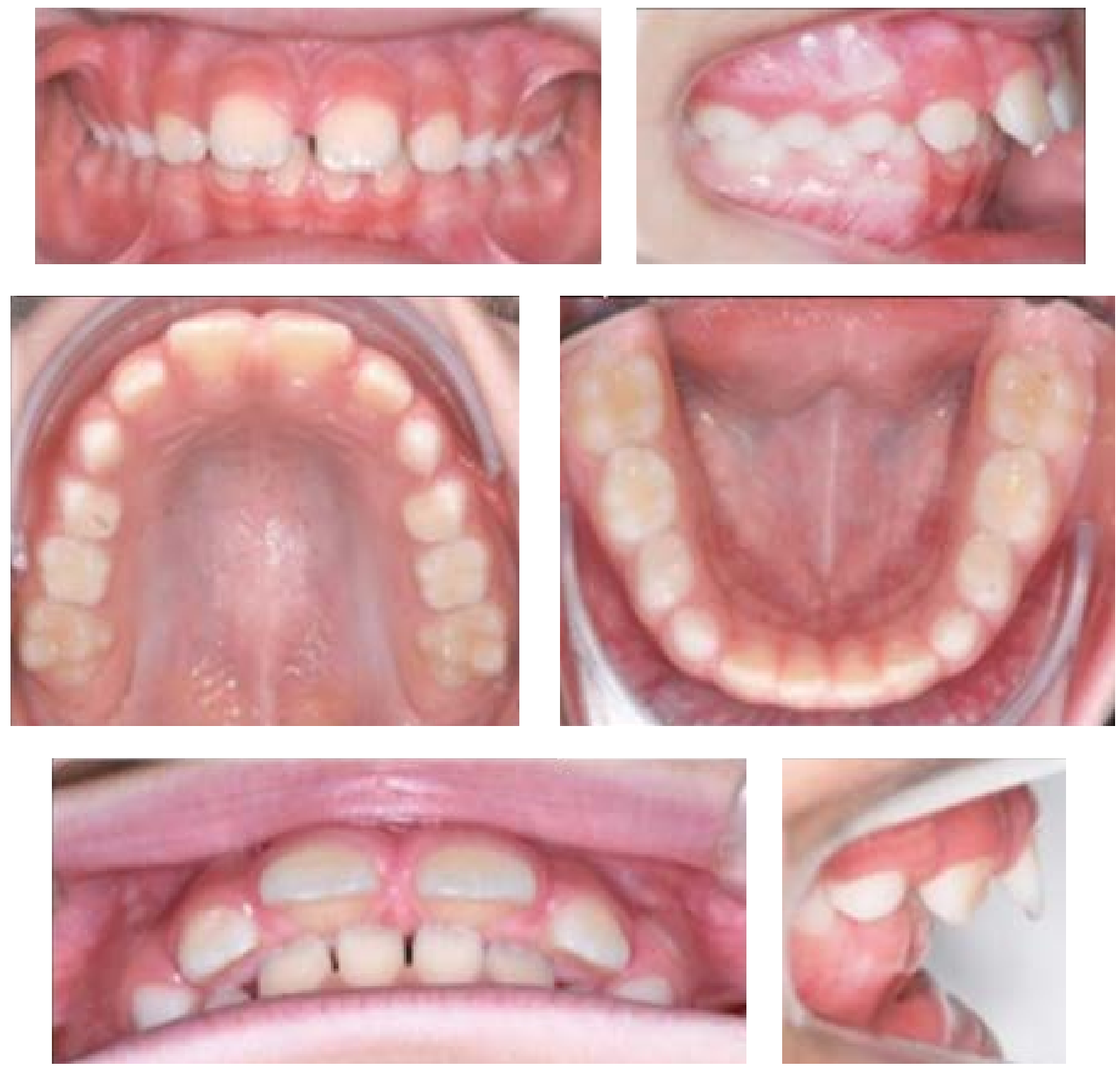

Figuras 27 a 32. Paciente B.B. Fotografías intraorales. 

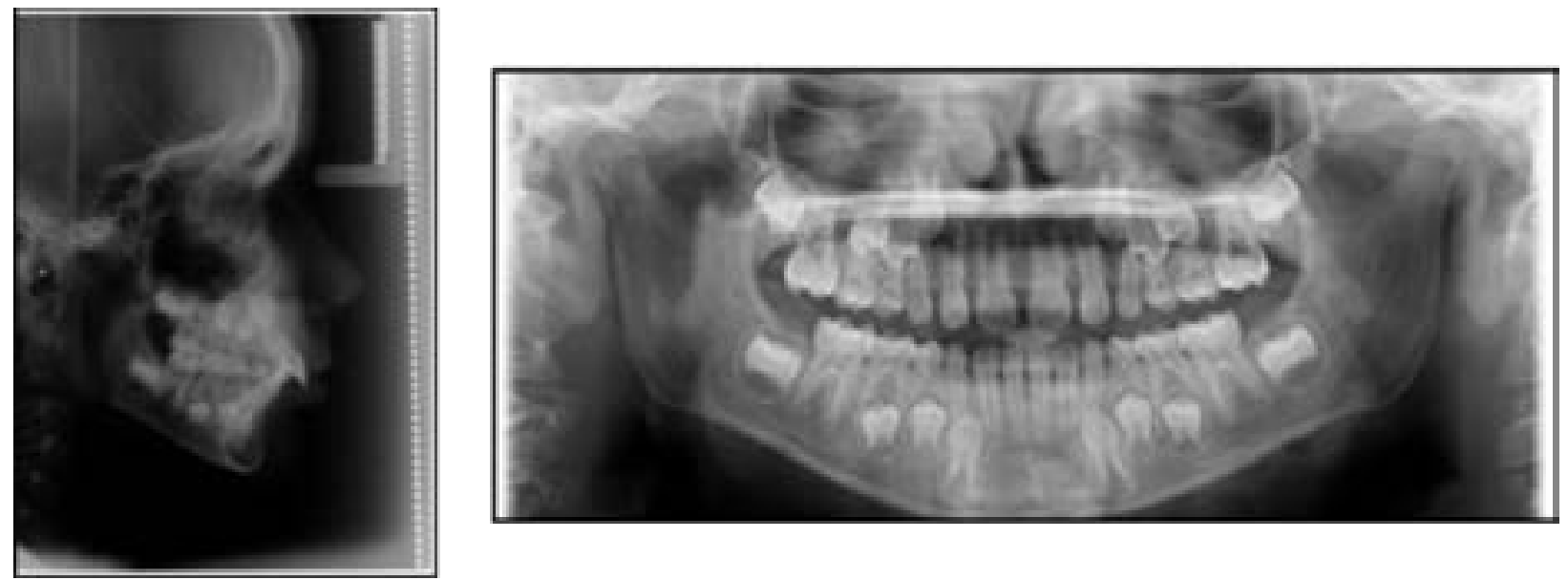

Figuras 33 y 34. Paciente B.B. Telerradiografía y O.P.T. iniciales.
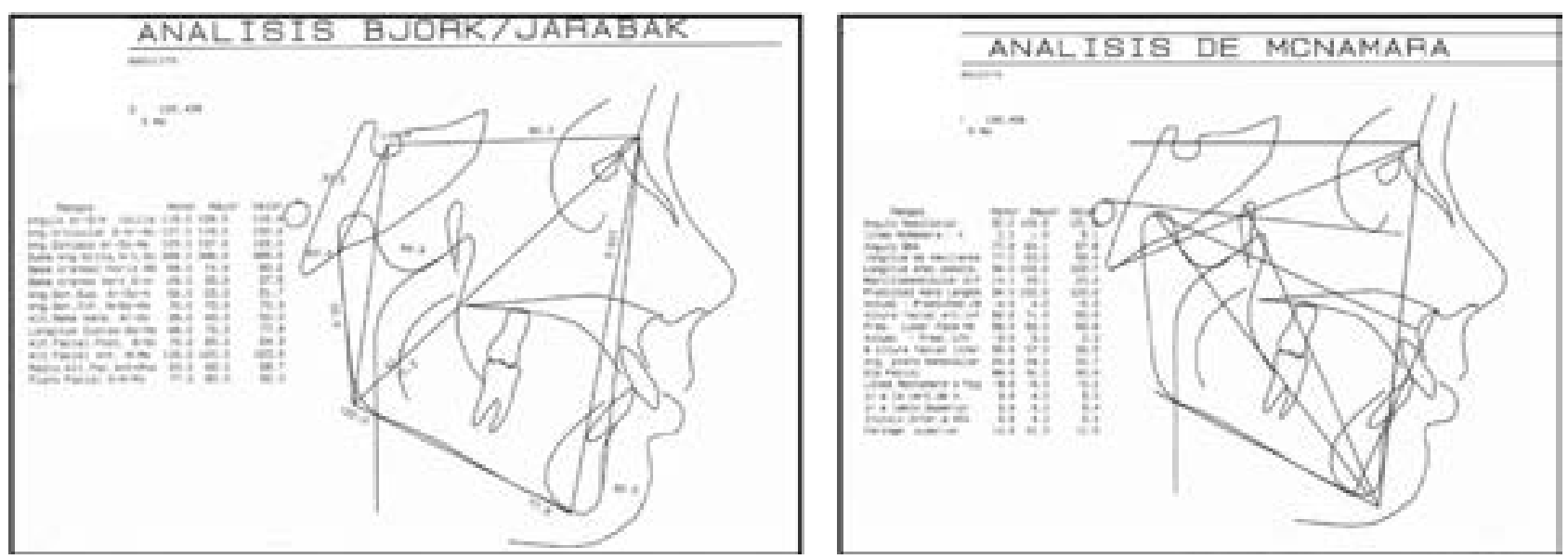

Figuras 35 y 36. Trazados Cefalométrico de Björk y McNamara.

\section{Plan de tratamiento}

Médico - Odontológicos: Derivación a otorrinolaringólogo para la evaluación de permeabilidad de vías respiratorias. Continuar reeducación de la deglución.

Odontopediatría: (Dra. Verónica Muguerza) Al momento de la fractura de pieza 11, se realizó colocación de Hidróxido de Calcio fraguable y resina compuesta de fotocurado. Control clínico y radiográfico de la pieza por un período de un 6 meses antes del comienzo del tratamiento con aparatología funcional y continúa por el plazo de un año y 6 meses más durante el cual se ha dado un cambio de terapéutica.
Se realiza control de placa bacteriana y enseñanza de cepillado con remotivación en todos sus controles con buenos resultados.

Ortodóncicos: Primera etapa - Estimular crecimiento de maxilar inferior en sentido sagital. Disminuir inclinación vestibular de incisivos superiores y vestibularizar los inferiores. Colaborar en la reeducación del labio inferior.

Segunda Etapa: Rotación de piezas 16 y 26. Intrusión (con fuerzas de muy baja intensidad) de incisivos, fundamentalmente inferiores. Continuar trabajando sobre el overjet. Cerrar diastemas. 
Al finalizar el recambio dentario, reevaluar y continuar con etapa de aparatología fija completa hasta alcanzar objetivos.

Medio terapéutico:

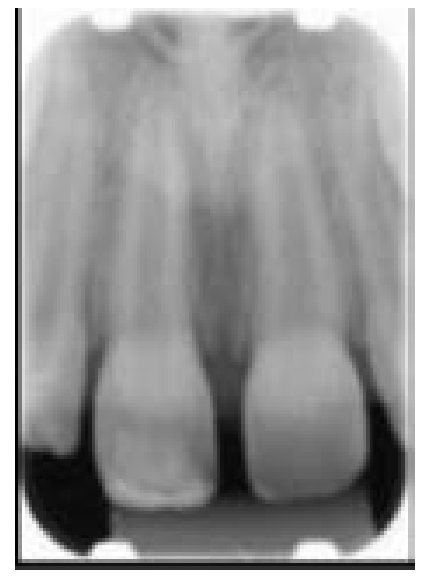

Figura 37. Radiografía periapical a 8 meses del traumatismo que muestra un correcto cierre de ápices radiculares.
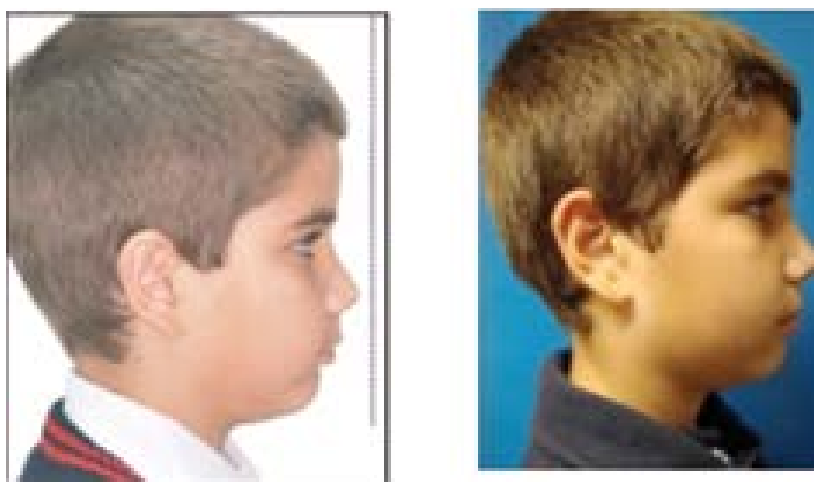

Figuras 40 y 41. Fotografías laterales, paciente B.B. antes $y$ después de 10 meses de tratamiento.
Primera etapa - Activador Abierto de Klammt con escudilos retrolabiales inferiores.

Segunda etapa - Aparatología fija, multibracket, Técnica de Arco Recto, prescripción Roth 0.022.
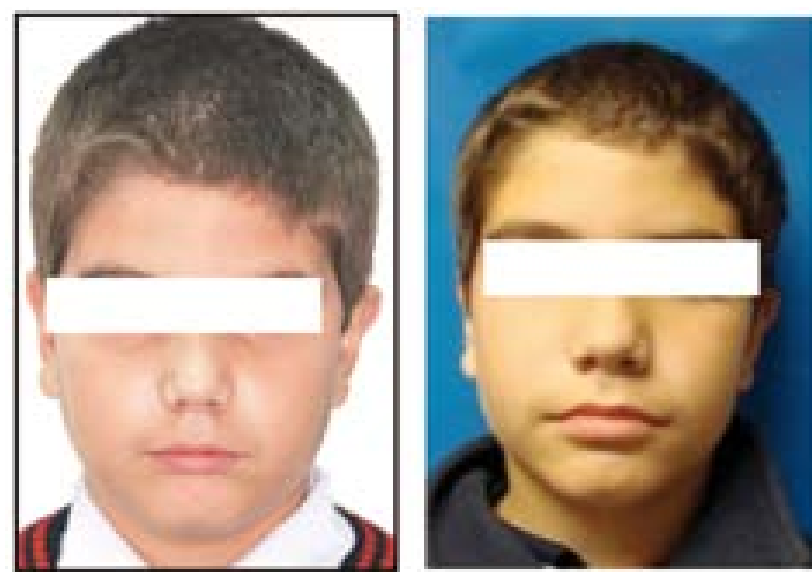

Figuras 38 y 39. Fotografías frontales de cara, paciente B.B. antes y después de 10 meses de tratamiento.

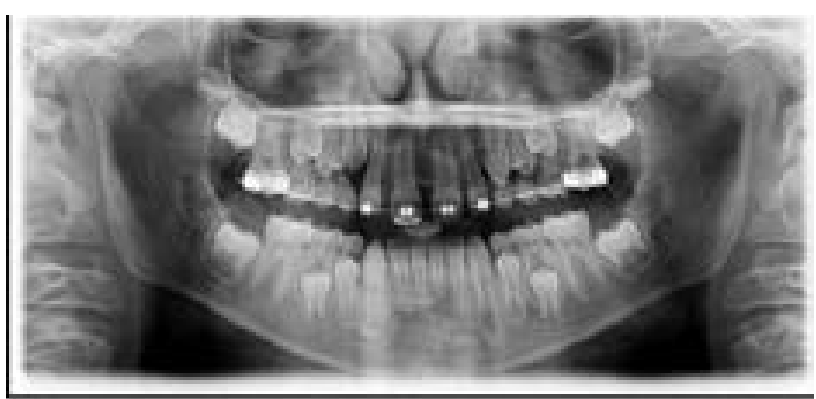

Figura 42. Ortopantomografía a los 18 meses, con Ap. Fijo en incisivos y molares 16 y $26(2 \times 4)$.
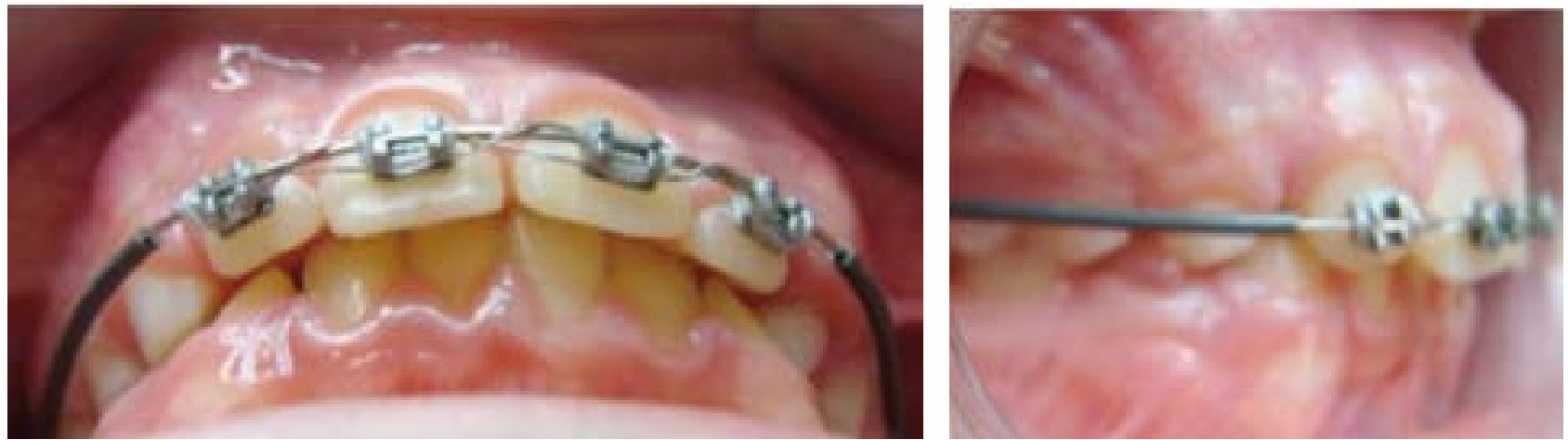

Figuras 43 y 44. Fotografía del sobrepase horizontal. 


\section{Discusión}

En ambos casos presentados se observa una atención con enfoque en el individuo y sus necesidades, desde un equipo de trabajo que resuelve en interconsultas cada aspecto de la situación problema, buscando óptimos resultados.

El primer caso, se trata de un paciente aprensivo, preocupado por su estética en plena etapa de la adolescencia, y que busca empatía en los profesionales tratantes (Odontopediatría: Dra. Verónica Muguerza, Ortodoncia: Dra. Annabel Grassi), brindándole seguridad y compartiendo con él la responsabilidad de una evolución favorable para su diente fracturado, para la resolución más adecuada de su disgnacia y para el logro y mantenimiento de su salud bucal y general. El segundo caso, se trata de un niño muy tímido y temeroso, en la edad en que la probabilidad de accidentes con traumatismos dentarios es mayor. Seguramente, el traumatismo podría y debería haber sido evitado tomando las medidas preventivas adecuadas, fundamentalmente a través del uso de protector bucal en prácticas deportivas. La inclusión del Licenciado en Fonoaudiología en el equipo de salud, suele ser muy útil en la derivación oportuna y recíproca cuando así se requiera, redundando en la optimización del tratamiento integral del paciente niño.
Los casos presentados, evidencian la importancia de la actuación de equipos interdisciplinarios y multiprofesionales.

\section{Conclusiones}

Los traumatismos dentoalveolares son eventos que ocurren frecuentemente en la niñez y adolescencia. Las estadísticas revelan cifras significativas en cuanto a la incidencia y prevalencia de estos accidentes, que nos obliga a considerarlos como un problema de salud pública global que puede y debe prevenirse con educación.

Es por ello que la Ortodoncia y la Ortopedia que son especialidades que tienen mucho para aportar en los casos de pacientes con traumatismos dentarios, ya sea en las etapas de diagnóstico, como de planificación de tratamiento y en el tratamiento propiamente dicho, deben contarse dentro del equipo multidisciplinario que trate dichos casos, fundamentalmente en servicios de salud. También, dada la incidencia de las maloclusiones en los traumatismos, estas disciplinas pueden brindar gran apoyo en la prevención de los mismos, mejorando las relaciones esqueléticas y dentarias que resultan ser factores de riesgo.

\section{Referencias}

1 - Glendor U., Marcenes W., Andreasen JO, Clasificación, Epidemiología y etiología. En: Andreasen J.O. et al. (Ed) Texto y Atlas a Color de Lesiones traumáticas a las Estructuras dentales. 4a Ed. T.1. Caracas : AMOLCA; 2010 pp.- $217-254$.

2 - Varela M. Ortodoncia y traumatología dental Cap. 7 En: Ortodoncia Interdisciplinar Vol. 1; Barcelona: Ed. Océano; 2005 pp.- 195 - 233

3 - Cisneros R.; Badanelli P.; Martínez Berná A. Traumatología dental: Aspectos generales. Cap 6 En: Ortodoncia Interdisciplinaria Vol. 1; Barcelona: Ed. Océano; 2005 pp.- 169 - 193

4 - Ribeiro de Rezende L.; Corrêa, Ma. S. Nahás P.; Almeida R. Traumatismo en la dentición decidua. En: Odontopediatría en la Primera Infancia. Corrêa Ma. S. Nahás P., San Pablo: Gen; 2009 p.p. 531 - 553

5 - International Association of Dental Traumatology. Dental Trauma Guidelines. Revides 2011. Disponible en: http:// www.iadt-dentaltrauma.org/ 
6 - Berman L.H.; Blanco L.; Cohen S., Manual Clínico de Traumatología Dental. Madrid: Elsevier; 2008 pp.- 1 - 11.

7 - García Ballesta C, Pérez Lajarín L, Castejón Navas I. Prevalencia y etiología de los traumatismos dentales: Una revisión. RCOE 2003; 8 (2):131-41.

8 - Malgren O., Malgren B. Manejo Ortodóntico de la dentición traumatizada. En: Andreasen J.O. et al. (Ed) Texto y Atlas a Color de Lesiones traumáticas a las Estructuras dentales. 4a Ed. T.2. Caracas : AMOLCA; 2010 pp.- 669-711.

9 - García - Godoy F.; García - Godoy F.; García - Godoy F.M. Primary theeth traumatics injuries at a private pediatric dental Center. Endod Dent. Traumatol 1987; 3 (3) pp.- 126 -9

10 - Paniza E.; López Ma. del C.; Urgencias en Odontopediatría. En: Cátedra de Odontopediatría, Fac. de Odontología; Odontopediatría Hoy; Montevideo 2002; pp.- 147 - 159.

11 - McDonald R.; Avery D.; Lynch Th. Tratamiento de traumatismos dentarios y de los tejidos de sostén en Odontología pediátrica y del adolescente. Buenos Aires; Ed. Médica Panamericana; 1990 GUIDELINES_Book.pdf

12 - Flores MT, Andersson L, Andreasen JO, Guidelines for the Management of traumatic dental injuries. III. Primary teeth. Dental Traumatology 2007; 23: 196-202

13 - Ranadelli; Dennis Lesiones deportivas y protectores bucales. En: Pinkham Odontología Pediátrica, 3a Ed. Mexico D.F. McGraw - Hill; 2001 p.p. 690 - 700

14 - Flores MT, Holan G, Borum M, Andreasen JO, Lesiones a la Dentición Primaria En: Andreasen J.O. et al. (Ed) Texto y Atlas a Color de Lesiones traumáticas a las Estructuras dentales. 4a Ed. T.1. Caracas : AMOLCA; 2010 pp.-516-539

15 - Flores MT, Andersson L, Andreasen JO, Guidelines for the Management of traumatic dental injuries. I. Fractures and luxations of permanent teeth. Dental Traumatology 2007; 23: 66-71

16 - Flores MT, Andersson L, Andreasen JO, Guidelines for the Management of traumatic dental injuries. II. Avulsion of permanente teeth. Dental Traumatology 2007; 23: 130-136

17 - Ebeleseder KA, Santler G, Glockner K, Hulla H, Pertl C, Quehenberger F. An analysis of 58 traumatically intruded and surgically extruded permanent teeth. Endod Dent Traumatol 2000; 16: 34-39

18 - Kaban L. Traumatismo facial II: Lesiones dentoalveolares y fracturas mandibulares en Cirugía bucal y máxilofacial en niños. México: Interamericana; 1992

19 - Sastre T, Carrascal J. Reabsorción Radicular Cap 8. En Ortodoncia Interdisciplinar Vol. 1; Barcelona: Ed. Océano; 2005. pp.- $235-270$.

Recibido: 22-09-2013

Aceptado: 17- 12- 2013

Correspondencia: Annabel S. Grassi Vassallo,Dr. Pablo de María 1561 ap. 502. Montevideo - URUGUAY,C.P. 11200,Tel.: +598 24017110. Email: angrassi@gmail.com 